\title{
Benign Secondary Hypertension
}

National Cancer Institute

\section{Source}

National Cancer Institute. Benign Secondary Hypertension. NCI Thesaurus. Code C3658.

Mild to moderate high blood pressure that is caused by an underlying medical condition. 